\title{
A LOW-DOSE LASER (DIODE LASER) APPLICATION REDUCES POCKET PERIODONTAL DEPTH AND CLINICAL ATTACHMENT LOSS
}

\author{
ROBERT LESSANG*, YUNIARTI SOEROSO, ALBERT JUANDA, SANDRA OLIVIA KUSWANDANI
}

Department of Periodontology, Faculty of Dentistry, Universitas Indonesia, Jakarta, Indonesia. Email: robertlessang@gmail.com

Received: 16 September 2017, Revised and Accepted: 3 October 2017

\section{ABSTRACT}

Objective: The initial therapy for chronic periodontitis is scaling and root planing (SRP), followed by non-surgical and surgical therapy. Recently, low-dose laser therapy was mentioned as an adjunctive therapy for periodontitis.

This study aims to analyze the effect of degranulation using a low-dose laser (diode laser) after SRP for chronic periodontitis treatment with an attachment loss of $\leq 5 \mathrm{~mm}$ compared to SRP alone.

Methods: A split-mouth design of in 158 tooth surfaces divided into two groups. The first group was treated with a low-dose laser (diode laser) after SRP, while the second group was treated with SRP only. Bleeding on probing (BOP), probing pocket depth (PPD), and clinical attachment loss (CAL) were all examined at baseline and 35 days later.

Results: The results were statistically significant differences between the two groups were indicated in terms of PPD ( $\mathrm{p}=0.000)$ and $\mathrm{CAL}(\mathrm{p}=0.040)$. No significant difference was identified in terms of BOP.

Conclusion: The application of a low-dose laser (diode laser) is effective in reducing PPD and CAL as an adjunct therapy in chronic periodontitis treatment.

Keywords: Periodontitis, A low-dose (diode) laser, Chronic periodontitis.

(c) 2017 The Authors. Published by Innovare Academic Sciences Pvt Ltd. This is an open access article under the CC BY license (http://creativecommons. org/licenses/by/4. 0/) DOI: http://dx.doi.org/10.22159/ijap.2017.v9s2.30

\section{INTRODUCTION}

Chronic periodontitis is a bacterial infection that causes inflammation of the periodontium, causing bone destruction and leading to tooth loss. The main bacteria in moderate and severe chronic periodontitis can be found through the bacterial cultures, such as Porphyromonas gingivalis, Prevotella intermedia, Tannerella forsythia, Treponema denticola, and Aggregobacter actinobacillus. The current new theory is that the specific bacteria, such as P. gingivalis, P. intermedia, T. forsythia, and $T$. denticola are closely related to an increase in probing pocket depth (PPD) and bleeding on probing (BOP) papilla bleeding index (PBI) $[1,2]$.

A common standard for the initial treatment of periodontal disease is subgingival instrumentation through scaling and root planing (SRP), which can be done manually or with additional instruments, such as ultrasonic equipment. Some studies have shown a good clinical outcome and microbial advantages. These include prevention against subgingival biofilm formation, a decrease in the number of bacteria, and the removal of plaque, calculus, and endotoxins [2,3]. According to the results, the clinical improvement following SRP is promising, but the bacteria remaining in the pocket can colonize and can be detected in a short time [2]. Clinical parameters for the successful periodontal treatment include oral hygiene score, plaque index, PBI, PPD, and clinical attachment loss (CAL) [4].

Two approaches can be taken in the treatment of periodontal disease progression: Surgical and non-surgical therapies. Surgical therapy can be achieved through curettage or flap surgery. In the past decade, nonsurgical therapy has focused on eliminating bacteria. Some treatment options have been exercised, such as the use of mouthwash, doxycycline and metronidazole, topical tetracycline and minocycline placed on the spherical cord, and low-dose tetracycline therapy twice a day for 90 days or more.
Currently, the use of a low-dose laser has been considered, as it aims to eliminate the bacteria from periodontal patient, it is easy to use a dentist in caring for and maintaining oral health [3,5]. Some researchers are trying to find alternative care options to reduce the use of antibiotics and other chemicals in the treatment of periodontitis, one of which is low-dose laser therapy.

Laser is an acronym for light amplification by stimulated emission of radiation. As is known, the main cause of periodontal disease is a bacterial infection, so eliminating periodontal pathogenic microorganisms will lead to positive outcomes. Toxins released from these bacteria will be destroyed by the laser's energy. SRP supplied with laser therapy has been shown to reduce pocket depth and improve oral health $[3,6]$. Several types of laser can be used for the treatment of periodontitis, such as a laser diode, erbium laser, Nd: YAG laser, CO2 laser, and others.

Ansersen et al. (2007), Kelbauskiene and Maciulskiene (2007), Ambrosini et al. (2005), and Neill and Mellonig (1997) examined the effectiveness of a photodynamic laser, erbium laser, and Nd: YAG laser against chronic periodontitis, and they argued that a laser can be used as an adjunct therapy to help improve clinical outcomes compared to SRP alone [7]. Lasers are a therapeutic option compared to curettage because the application time is faster; there is a better visualization, reduced pain, and increased patient comfort; it is less tiresome for the operator; and fewer special skills are required [8]. Some studies also found that curettage or degranulation with a granulation tissue laser will clean better than manual instrumentation [9].

Some authors found that using a laser diode after SRP was an excellent option in the treatment of chronic periodontal disease. Research on the effectiveness of laser diodes in conjunction with SRP in the treatment of chronic periodontitis has not been conducted in Indonesia; even across the world, fewer research articles were found [9]. Another advantage of the diode laser is its price, which is lower than other lasers [7]. 


\section{MATERIALS AND METHODS}

\section{Study design}

This study was performed as a randomized, split-mouth clinical trial. It was conducted from December 2012 to February 2013, and it included patients with a diagnosis of localized chronic periodontitis with a loss of attachment of $\leq 5 \mathrm{~mm}$ and BOP. All patients were informed of the procedures, purpose, duration, and outlines of the study, and they signed a written informed consent form before the study and approved by the Ethics Committee of the Faculty of Dentistry, Universitas Indonesia. The sample size was at least 24 teeth per group, and this resulted in a power of $95 \%$ to detect mean differences in CAL between the groups.

\section{Study population}

The study population consisted of 79 teeth surfaces in each group from 10 patients with a mean age of $35-55$ years. The patients were divided into two groups. The first group was a trial group including patients with chronic periodontitis localized on one tooth surface, a CAL of $\leq 5 \mathrm{~mm}$, and PBI, and they were treated with SRP and laser therapy. The second group was a control group including patients with chronic periodontitis localized on one tooth surface, a CAL of $\leq 5 \mathrm{~mm}$, and PBI, and they were treated with SRP alone.

\section{Patient selection criteria}

Research subjects were selected using the following inclusion criteria: Patients with localized chronic periodontitis with the teeth on one surface, a CAL of $\leq 5 \mathrm{~mm}$, PBI, at least 20 original teeth in the mouth (third molars are not counted), and aged 30-55 years. The study also included patients who did not consume antibiotics in the past 3 months, who have not undergone periodontal treatment in the past 6 months, and who are in good general health.

The following exclusion criteria were used: Patients with the presence of proximal fillings, infrabony pocket, proximal and cervical caries, and the existence of rampant caries and dental malocclusion. In addition, patients whose pregnancy, lactation, and physical conditions might hinder instrumentation, who consumed drugs moderately, who smoke more than 20 cigarettes per day, and who have the inability to maintain their oral hygiene were also excluded from the study.

\section{Clinical measurements}

To measure BOP, the PBI parameter introduced by Muhlmann was used, which is an examination of the presence/absence of bleeding in the proximal region. The mesial surface was measured from the labial/buccal region, while the distal surface was measured from the palatal/lingual region. PBI measurements on the mesial surface were taken by inserting a probe caution as deep as $1 \mathrm{~mm}$ into the bottom of mesial gingival sulcus, and the experiment was then shifted to the top of papillae and from the distal section adjacent to the top of the buccal gingival sulcus.

Bleeding intensity was measured for 20-30 seconds using the following scale: $0=$ no bleeding; 1 =bleeding, such as bleeding in the form of a dot; $2=$ bleeding in the form of a line; 3 =bleeding in the form of a triangle; and 4=bleeding spreads. PPD is a measurement of the periodontal pocket depth measured from the edge of the gingiva to the bottom of the pocket.

The CAL is measured with a periodontal probe with a millimeter scale. Increased tissue attachment loss is measured as the difference between before and after treatment

\section{Treatment protocols}

If trauma occlusion was found in the subjects, occlusion adjustments were made in advance as part of the initial therapy, and when there is a tooth mobility of $3^{\circ}$, splinting was applied beforehand and evaluated 1 week after the traumatic occlusion treatment. On the subject of the research is only done SRP, followed by measuring scores BOP, pocket depth, and attachment loss.
This measurement is calculated as day to 0 . On the day 0 , patients with chronic periodontitis have a pocket between 3 and $5 \mathrm{~mm}$ and an attachment loss $\geq 5 \mathrm{~mm}$ contralateral to each gear position. In the study area, scaling and root smoothing is conducted, followed by laser therapy applied to the pockets and the control area with SRP only. On the day 35, bleeding, pocket depth, plaque index, and attachment loss were measured.

\section{RESULT}

This research was conducted in the Periodontology Clinic of Dental Hospital, Universitas Indonesia. The study population consisted of 10 patients, with 79 teeth surfaces in the intervention group and 79 teeth surfaces in the control group. The result shows the distribution of the mean and an analysis of PBI, PPD, CAL, and the mean difference before and after treatment with SRP and laser therapy or SRP alone.

Table 1 shows the distribution of the mean and the analysis of PBI, PPD, and CAL before and after treatment with SRP and laser therapy or SRP alone. The data explain a reduction in the index of BOP after treatment with SRP and laser therapy and with SRP alone. The Wilcoxon test result shows a significant difference with a $p=0.00$. Table 1 shows the distribution of the mean and the analysis of PBI, PPD, and CAL before and after treatment with SRP and laser therapy or with SRP alone.

The data explain that there is a reduction in the PBI and PPD after treatment with SRP and laser therapy and with SRP alone.

The statistical Wilcoxon test result for PBI and PPD shows a significant difference with a $\mathrm{p}=0.00$. The statistical Mann-Whitney test result for CAL shows no significant difference with $\mathrm{p}=0.968$ for SRP alone and $\mathrm{p}=0.603$ for SRP+Laser. Table 2 shows the distribution analysis of the mean difference before and after treatment with SRP and laser therapy or with SRP alone. The data explain that there is a significant difference in the PPD index $(p=0.000)$ and CAL $(p=0.040)$, but there was no significant difference in the BOP index $(\mathrm{p}=0.082)$

\section{DISCUSSION}

This research aimed to investigate the degranulation effect with a diode laser after SRP on chronic periodontitis with an attachment loss of $\leq 5 \mathrm{~mm}$ compared to SRP alone. Laser degranulation is a localized, antiseptic treatment, and it can be used as a surgical and antibiotic therapy that has a standard for chronic periodontitis treatment. Clinical parameters include the PBI, PPD, and CAL $[10,11]$. A sample size of 158 teeth with 79 teeth surfaces in the intervention group (SRP and laser therapy) and 79 teeth surfaces in the control group (SRP alone) was used. Research subjects were chosen following the criteria of an age over 30 years with gingival inflammation, periodontal pockets, and attachment loss.

Clinically, PBI improved in each group from baseline until follow-up day. From the data, we can see the differences between the intervention and control groups clinically, but the differences are not statistically significant $(p>0.05)$. This research result agrees with the findings of Birang et al., who showed no significant difference in terms of BOP, but Aykol et al.'s study found a contrary result [11].

PPD improved in each group from baseline until follow-up day in clinical and statistical. From the data, we can see the differences in the result between the intervention and control groups clinically and statistically $(p<0.05)$. This result is in line with the theory that laser therapy can remove granulation tissues in the pocket wall [12].

CAL categories, from the data, we can see the differences in the results between the intervention and control groups are statistically significant but not significant in clinical appearance. Research from Rodrigues et al. (2014), which applied a diode laser as an adjunct therapy for chronic periodontitis, did not produce a significant result 
Table 1: Distribution of the mean and the analysis of PBI before and after treatment with SRP and laser therapy or with SRP alone

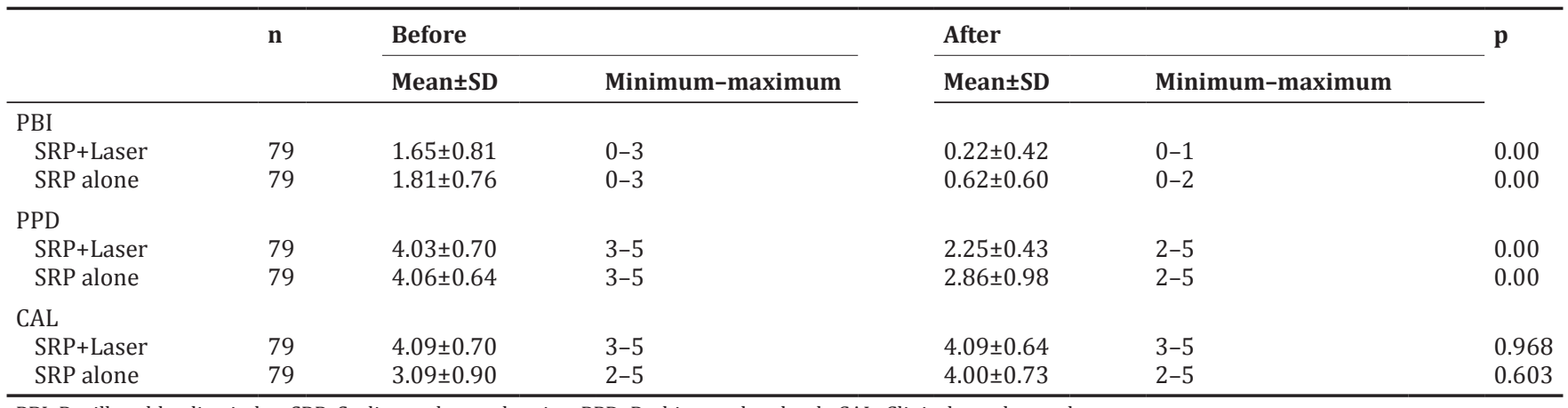

PBI: Papillary bleeding index, SRP: Scaling and root planning, PPD: Probing pocket depth, CAL: Clinical attachment loss

Table 2: Distribution and analysis of the mean difference of SRP and laser therapy or SRP alone

\begin{tabular}{lclll}
\hline Variables & $\mathbf{n}$ & \multicolumn{2}{c}{ Mean difference } & \multirow{2}{*}{$\mathbf{p}$} \\
\cline { 3 - 4 } & & Mean \pm SD & Minimum-maximum & \\
\hline PBI & & & & 0.082 \\
$\quad$ SRP+LASER & 79 & $1.43 \pm 0.84$ & $0.00-3.00$ & \\
$\quad$ SRP alone & 79 & $1.18 \pm 0.78$ & $1.00-3.00$ & \multirow{2}{*}{$0.000^{*}$} \\
PPD & & & & \\
$\quad$ SRP+LASER & 79 & $1.78 \pm 0.72$ & $0.00-3.00$ & $0.040^{*}$ \\
SRP alone & 79 & $1.20 \pm 0.88$ & $0.00-3.00$ & \\
CAL & & & & \\
$\quad$ SRP+LASER & 79 & $0.18 \pm 0.39$ & $0.00-1.00$ & \\
SRP alone & 79 & $0.08 \pm 0.32$ & $0.00-2.00$ & \\
\hline
\end{tabular}

SRP: Scaling and root planning, PBI: Papillary bleeding index, PPD: Probing pocket depth, CAL: Clinical attachment loss

in reducing the CA [13]. This result may be related to the follow-up interval time. Sharaf et al. (2012) stated that SRP with diode laser therapy was more effective than SRP alone in the chosen patients, and the antibacterial effect continued to be significant up to 6 weeks after therapy [14].

In this result, we found that diode laser therapy, as an adjunct therapy in chronic periodontitis patients, is effective in reducing PPD and CAL. This result could lead to the recommendation of using laser therapy as an initial treatment to support SRP treatment. The main functions of laser therapy include relieving the epithelial surface and periodontal pocket disinfection. Research from Gupta (2016) also showed the effectiveness of diode laser therapy in removing A. actinomycetemcomitans from the periodontal pocket, and this can be attributed to the destruction of critical virulence factors (lipopolysaccharide and proteases) present in the bacteria.

The wavelength of the diode laser is $800-980 \mathrm{~nm}$, which can be absorbed into hemoglobin and other pigments. The pigments of the anaerobic periodontitis pathogen lead to the vaporization of water, and this could damage the bacterial cell wall until lysis and death [15].

Laser therapy is not a substitute for the main treatment to eliminate bacteria in dental plaque and calculus as a main etiology of inflammation causing periodontal disease. The use of a diode laser can lead to side effects from the heat produced from the laser, because it is a blind technique, so dentists cannot see the depth of penetration. Avoiding this situation, knowledge about the optimum power of the laser in degranulation therapy is needed [16].

Regarding limitations of the study, future research must develop longterm longitudinal research to determine the stabilization of laser treatment and the cost-effectiveness of using a diode laser as adjunct therapy for chronic periodontitis patients to elevate the advantages of laser therapy as simple, painless, and comfortable for patients. In the future studies, research using bacteria and biomoleculer analyses can enhance the results of the study.

\section{CONCLUSION}

Laser therapy as an adjunct treatment for chronic periodontitis patients has preeminence to eliminate bacteria in periodontal pockets. This study has concluded that in terms of PPD and CAL the treatment of chronic periodontitis patients with SRP and laser therapy has significant differences in terms of statistics from treatment with SRP alone. Contrarily, in other variables, treatment with SRP and laser therapy has the same effect as SRP treatment alone.

\section{ACKNOWLEDGMENT}

The publication of this manuscript is supported by Universitas Indonesia.

\section{REFERENCE}

1. Hujoel P, Zina LG, Cunha-Cruz J, Lopez R. Historical perspectives on theories of periodontal disease etiology. Periodontol 2000 2012;58:153-60.

2. Birang R, Shahaboui M, Kiani S, Shadmehr E, Naghsh N. Effect of Nonsurgical Periodontal Treatment Combined With Diode Laser or Photodynamic Therapy on Chronic Periodontitis: A Randomized Controlled Split-Mouth Clinical Trial. J Lasers Med Sci 2015;6:112-9.

3. Parker S. Lasers and soft tissue: Periodontal therapy. Br Dent J 2007:202:309-15.

4. Goel K, Baral D. A Comparison of impact of chronic periodontal diseases and nonsurgical periodontal therapy on oral health-related quality of life. Int J Dent 2017;2017:9352562.

5. Feitosa. Use of the diode laser in the nonsurgical treatment of periodontal pockets. Odontol Clin Sci 2007;6:129-32.

6. American Academy of Periodontology statement in the efficacy of lasers in the non-surgical treatment of inflamatory periodontal disease. J Periodontol 2011;82:513-4.

7. Karlsson MR, Diogo Löfgren CI, Jansson HM. The effect of laser therapy as an adjunct to non-surgical periodontal treatment in subjects with chronic periodontitis: A systematic review. J Periodontol 2008;79:2021-8.

8. Lin J, Bi L, Wang L, Song Y, Ma W, Jensen S, et al. Gingival curettage study comparing a laser treatment to hand instruments. Lasers Med Sci 2011;26:7-11.

9. Lagdive S, Marawar P, Mani AM. Semiconductor diode lasers in periodontics: An innovative and safe. Int J Appl Basic Med Res 2011;1:66-70.

10. Prabhu A, Rajbhoj S, Anjankar J, Maknojia M. Clinical applications of diode laser in periodontal therapy-a case series. IOSR J Dent Med Sci 2016;15:12-6

11. Aykol G, Baser U, Maden I, Kazak Z, Onan U, Tanrikulu-Kucuk S, et al. The effect of low-level laser therapy as an adjunct to non-surgical periodontal treatment. J Periodontol 2011;82:481-8.

12. Goldstep F, Freedman G. Diode lasers for periodontal treatment. Dent Asia 2011;???:20-3.

13. Rodrigues SV, Rane TS, Vijayakar HN, Mitra DK, Shah RA, Pathare PN. Use of $810 \mathrm{~nm}$ diode laser as an adjunctive therapy in 
chronic periodontitis: A randomized controlled clinical trial. J Indian Dent Assoc 2014;8:8-12.

14. Sharaf HM, Elkhodary AM, Saafan AE, Mostafa MI, El Hadidy SM, ElShenawy HM. Antibacterial effectiveness of low energy diode laser irradiation on management of periodontitis in down syndrome. Eur $\mathbf{J}$ Hum Mov 2012;47:226-37.
15. Gupta S, Sawhney A, Jain G, Dhar S, Gupta B, Singh R, et al. An evaluation of diode laser as an adjunct to scaling and root planning in the nonsurgical treatment of chronic periodontitis: A clinicomicrobiological study. Dent Med Res 2016;4:44-9.

16. Pirnat S. Versality of an $810 \mathrm{~nm}$ diode laser in dentistry: An overview. J Laser Health Acad 2007;4:1-9. 